Ann. Biol. anim. Bioch. Biophys., 1978, 18 (4), 773-780.

\title{
Etude par radioimmunologie des propriétés des immunsérums de I'hormone gonadotrope de la carpe (Cyprinus carpio) et de ses sous-unités
}

\author{
par Elisabeth BURZAWA-GÉRARD, B. KERDELHUÉ * \\ avec la collaboration technique de Michèle VALLENS, Yvette LANGLOIS, Aléna DUMAS \\ Laboratoire de Physiologie générale et comparée du Muséum national d'Histoire naturelle, \\ Laboratoire d'Endocrinologie comparée associé au C. N. R. S., 7 rue Cuvier, 75005 Paris \\ * Laboratoire des Hormones polypeptidiques, C. N. R. S., 91190 Gif-sur-Yvette.
}

Summary. Radioimmunological properties of antisera from carp gonadotropin (c-GTH) and its subunits.

Anti-sera against carp gonadotropin (c-GTH) and its subunits SU I and SU II were produced in rabbits (IS-c-GTH, IS-SU I and IS-SU II). The radioimmunological titers were determined when antiserum binding dilution was 50 p. 100 of the ${ }^{125}$-labelled glycoproteins. The titers of IS-c-GTH were $1 / 750000,1 / 300000$ and $1 / 500000$, respectively with c-GTH, SU I and SU II. With SU I, the titer of IS-SU I was $1 / 100000$; this antiserum in excess bound only 25 p. 100 of the c-GTH and no SU II. With c-GTH and SU II, the titer of IS-SU II was $1 / 270000$; it was $1 / 13000$ with SU I. With these antisera no binding was observed with rat hormones (LH, FSH, TSH, PRL), but IS-c-GTH and IS-SU II weakly bound $\mathrm{rLH}$. Displacement studies were also carried out ; the cross-reactions in each system were as follows :

IS-c-GTH ${ }^{125}$ I-c-GTH 100 p. 100 with SU II (for $0.1 \mathrm{ng}$ ) 0.01 p. 100 with SU I

IS-SU I ${ }^{125}$ I-SUI 20 p. 100 with c-GTH (for $0.1 \mathrm{ng}$ ) 0 p. 100 with SU II

IS-SU II ${ }^{125}$ I-SU II 25 p. 100 with c-GTH 0 p. 100 with SU I.

No displacement was observed in any test with rat hormones, even at high doses $(100 \mathrm{ng})$. c-GTH and its subunits were very antigenic in rabbit and thus we could separately test each subunit.

L'hormone gonadotrope de la carpe (c-GTH) est composée comme toutes les hormones hypophysaires glycoprotéiques de deux sous-unités. Cette hormone, ainsi que ses sous-unités (SU I et SU II), ont été obtenues à un degré élevé de pureté (Burzawa-Gérard, 1971, 1974). Toutefois, il existe un équilibre entre l'hormone et ses sousunités et de ce fait toutes les préparations de c-GTH contiennent des sous-unités libres en faible proportion (Burzawa-Gérard, 1974).

Les méthodes de dosages radioimmunologiques décrites jusqu'à présent mettent en jeu la GTH de carpe (Breton ef al., 1971, 1972, 1973) ou celle d'un saumon (CRIM et al., 1973). Des résultats importants ont pu être apportés en ce qui concerne la varia- 
tion des taux plasmatiques en GTH au cours des cycles de reproduction du saumon ou du cyprin. Toutefois, il est probable, fout au moins en ce qui concerne la c-GTH, que des systèmes assez complexes aient été mis en jeu. En effet, l'hormone marquée peut également contenir des sous-unités marquées et les immunsérums des anticorps, non seulement contre l'hormone, mais aussi contre ses sous-unités.

Il apparaît donc nécessaire de préciser les propriétés radioimmunologiques des sous-unités comparées à celles de l'hormone. Certains résultats préliminaires ont été brièvement décrits (Burzawa-Gérard et al., 1977).

\section{Matériel et méthodes.}

a) Hormones utilisées. La c-GTH et ses sous-unités sont obtenues selon les techniques précédemment décrites (Burzawa-Gérard, 1971, 1974). Les hormones gonadotropes de rat ( $r-\mathrm{LH}, r-\mathrm{FSH})$ sont préparées par Jutisz et Ribot ; l'hormone thyréotrope de Rat $(r-T S H)$ et la prolactine de Rat ( $r-P R L)$ sont fournies par le NIAMD.

b) Techniques radioimmunologiques. Les immunsérums anti-c-GTH (IS-c-GTH) anti SU I (IS-SU I) et anti SU II (IS-SU II) ont été obtenus chez le lapin après 4 injections de c-GTH ou 5 injections des sous-unités émulsionnées dans l'adjuvant de Freund complet. Les immunsérums obtenus sont gardés congelés ou lyophylisés jusqu'au moment de leur utilisation (Kerdelhué et al., 1971).

La C-GTH, SU I et SU II sont marquées à l'iode ( ${ }^{125}$ ) par la chloramine T selon la technique de Greenwood et al., (1963) dans les conditions utilisées dans le cas des hormones gonadotropes de rat (Kerdelhué et al., 1971). Une filtration sur Sephadex G 75 permet d'éliminer l'iode libre des glycoprotéines iodées (seule sera utilisée la fraction correspondant au maximum de la radioactivité). L'activité spécifique des solutions marquées est d'environ 100 à $150 \mu \mathrm{C} / \mathrm{mg}$.

La réaction radioimmunologique est réalisée en tampon phosphate $(0,1 \mathrm{M}, \mathrm{pH}$ $7,4)$ contenant de l'albumine sérique de bouf $(0,1$ p. 100$)$ dans un volume final de $520 \mu \mathrm{l}$. Les essais sont réalisés en triplicas et incubés $48 \mathrm{~h}$ à $4{ }^{\circ} \mathrm{C}$. Le complexe hormoneanticorps est isolé par immunoprécipitation ; dans ce but on ajoute du sérum de mouton anti- $\gamma$-globuline de lapin ef on incube $24 \mathrm{~h}$ à $4^{\circ} \mathrm{C}$. Le précipité formé est séparé par centrifugation (1000 g) et le surnageant éliminé par aspiration. La radioactivité du précipité est déterminée par un compteur $\gamma$ en nombre de coups par mn (cpm).

c) Techniques biochimiques. L'analyse des produits iodés est réalisée par filtration sur Sephadex G 100 et par électrophorèse analytique sur gel de polyacrylamide.

\section{Résultats.}

A. Marquage de la c-GTH et de ses sous-unités.

La filtration sur Sephadex G 100 d'une fraction de c-GTH marquée montre la présence de deux pics. Le premier correspond au coefficient d'exclusion de l'hormone $\left(K_{D} \simeq 0,30\right)$, le second $K_{D} \simeq 0,45$ n'est pas différent de celui des sous-unités (fig. 1 ). Les sous-unités SU I et SU II marquées sont elles-mêmes exclues pour un coefficient d'exclusion $\left(K_{D} \simeq 045\right)$ voisin de celui des sous-unités natives (fig. 1$)$. Ces résultats 
ont été confirmés par électrophorèse analytique sur gel de polyacrylamide. La proportion des sous-unités présentes dans les solutions de c-GTH marquées est augmentée.

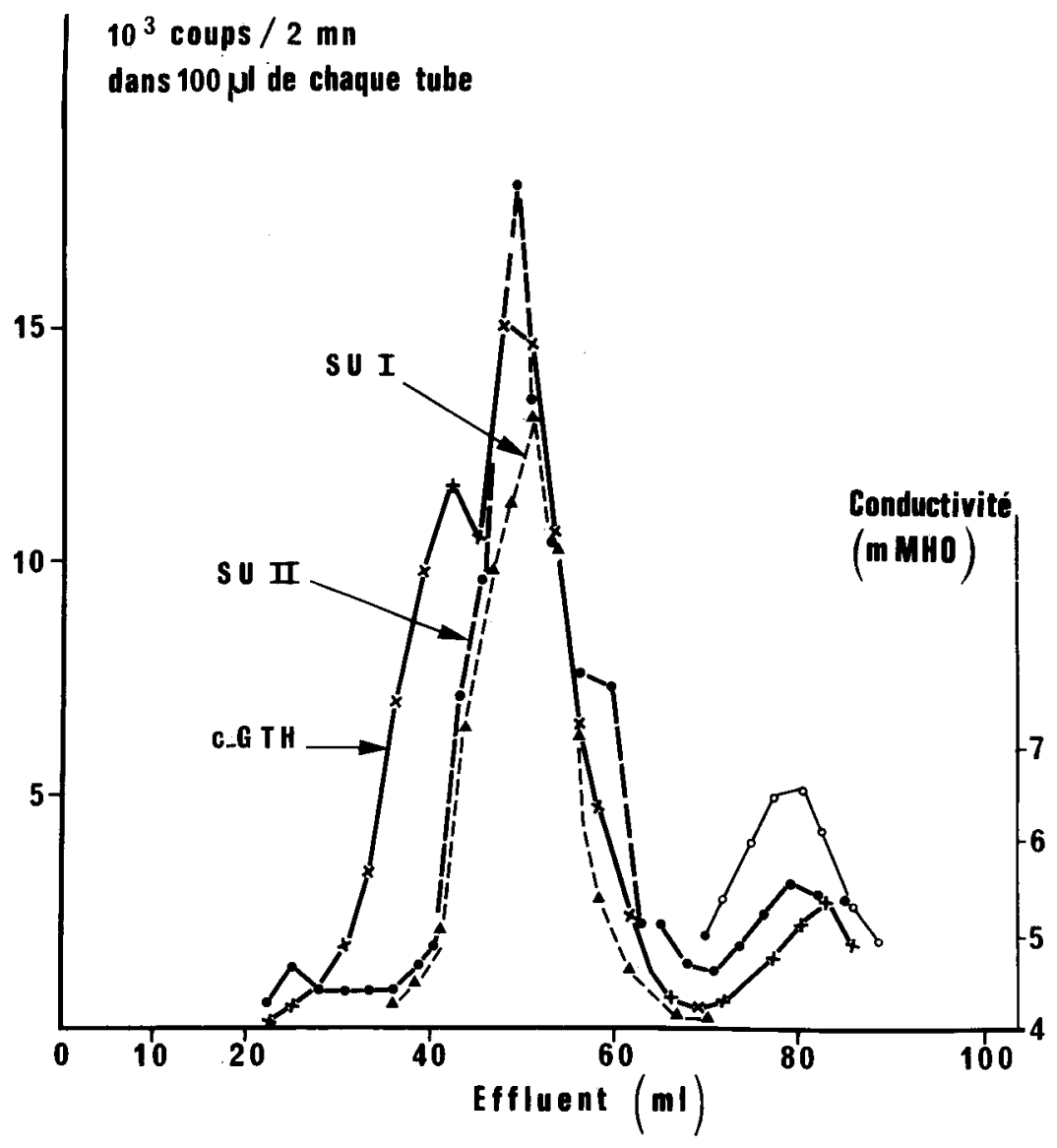

FIG. 1. - Efude par filtration sur Sephadex $G 100$ des solutions de c-GTH SU I ef SU II marquées obtenues après Sephadex $G 75$.

Colonne $1,2 \times 70 \mathrm{~cm}$; débit $17 \mathrm{ml} / \mathrm{h} \mathrm{1,6} \mathrm{ml/fraction.} \mathrm{Tampon} \mathrm{tris} \mathrm{H}-\mathrm{Cl} 0,05 \mathrm{M}, \mathrm{pH} 7,7$.

$10 \mu l$ de chaque solution radioactive sont dilués dans $1 \mathrm{ml}$ de $\mathrm{NaCl} 0,5 \mathrm{M}$, auxquels sont ajoutés $1 \mathrm{mg}$ de c-GTH, SU I ou SU II selon qu'il s'agit de ${ }^{125}$ I-c-GTH, ${ }^{125}$ I-SU I ou ${ }^{125}$ I-SU II.

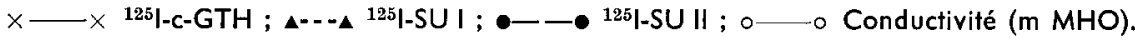

B. Ełude des immunsérums anti c-GTH et anti sous-unités.

a) Le titre radioimmunologique est représenté par la dilution finale de l'immunsérum considéré qui permet d'obtenir 50 p. 100 de liaison avec l'antigène marqué. Les titres de l'IS-c-GTH sont respectivement de $1 / 750000,1 / 300000$ et $1 / 500000$ avec c-GTH, SU I ef SU II (fig. 2). Le titre de I'IS-SU I vis-à-vis de SU I est de 1/1 000000 par contre il n'y a pas de titre définissable pour la c-GTH et SU II. L'IS-SU I en excès ne 


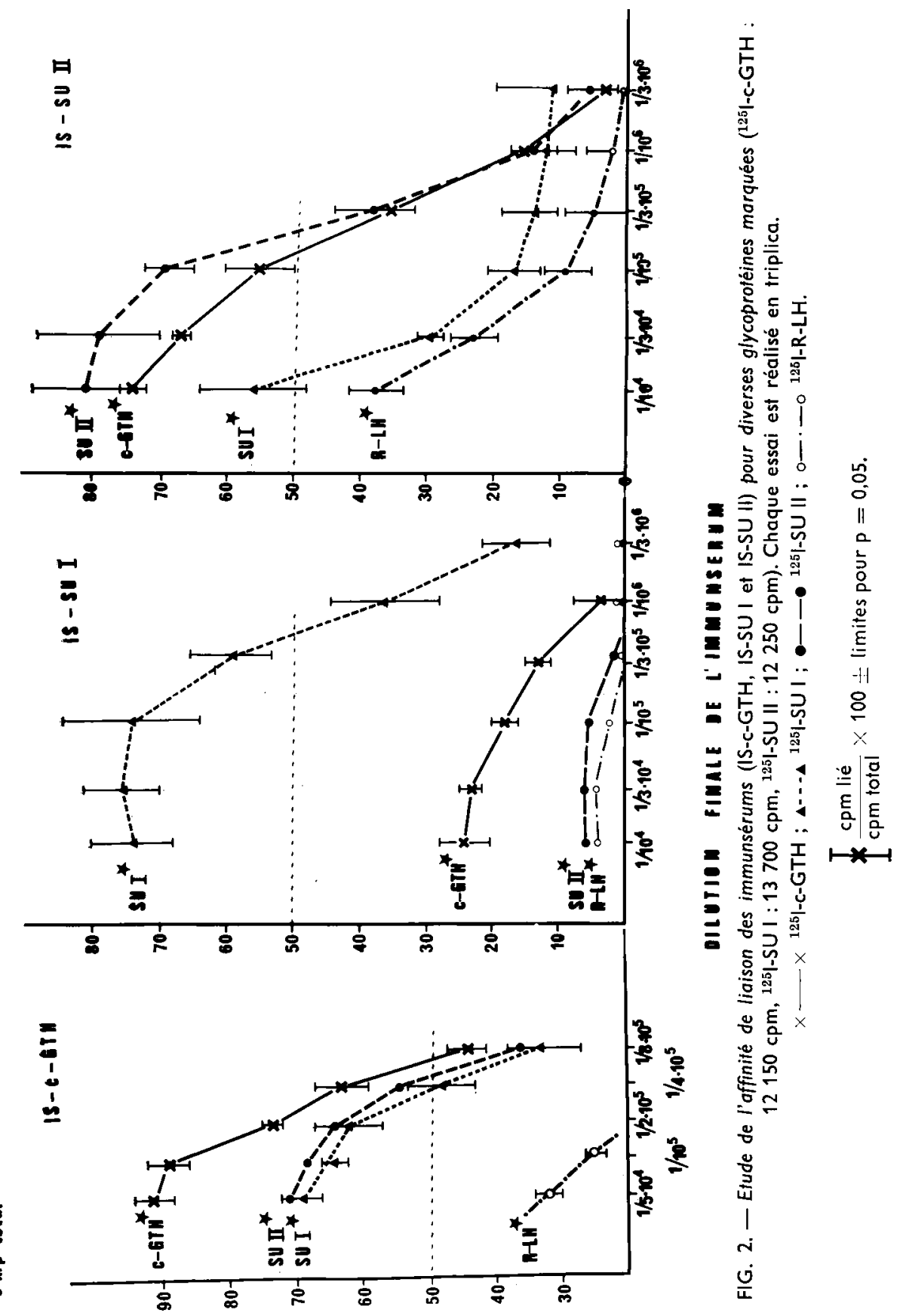


lie que 25 p. 100 de la c-GTH et pratiquement pas SU II (fig. 2). Les titres de l'IS-SU II vis-à-vis de la c-GTH ef de SU II ne sont pas sensiblement différents $1 / 270000$; vis-àvis de SU I le titre est de $1 / 13000$ (fig. 2). Aucun de ces trois immunsérums ne possède de sites de liaison pour la r-FSH, la r-TSH, mais les immunsérums IS-c-GTH et IS-SU II lient faiblement la r-LH (fig. 2).

b) Le déplacement de la liaison de l'antigène marqué à l'immunsérum correspondant a été étudié pour chacun des trois systèmes à l'aide de l'antigène lui-même ou d'autres glycoprotéines (hormone ou sous-unités et hormones hypophysaires de rat). Dans chaque cas la dilution de l'immunsérum entraînant 50 p. 100 de liaison de l'anłigène marqué a été utilisée.

1. Système IS-c-GTH ${ }^{125}$ I-c-GTH (fig. 3). Le pourcentage de liaison en l'absence d'hormone non marquée est de 49,3 $(46,3-52,3)$, il est de $37,3(36,2-38,4)$ pour $0,01 \mathrm{ng}$ de c-GTH. La sensibilité de ce système est donc inférieure à $10 \mathrm{pg}$. SU II déplace l'équilibre de ce système, mais de manière non parallèle à c-GTH (covariance entre les droites obfenues après la transformation Logit-Log (dose) pour la c-GTH marquée et la SU II marquée : $\left.F_{\text {cov. }}=14,99(\mathrm{dl}=1-18) \mathrm{p}<0,01\right)$. Mais la réaction croisée est pratiquement nulle arec SU I (0,01 p. 100).

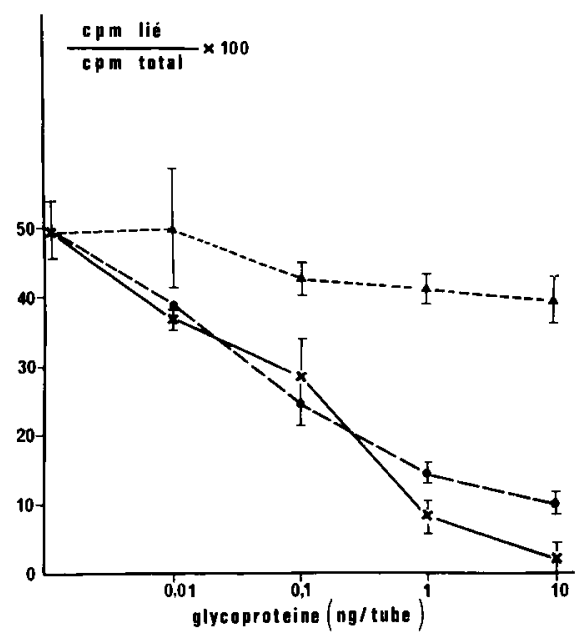

FIG. 3. - Déplacement de la liaison de ${ }^{125}$ I-C-GTH à I'IS-C-GTH par C-GTH, SU I ef SU II. Chaque essai est réalisé en triplica. IS-c-GTH dilution finale de 1/500 000. 125/-c-GTH : $12150 \mathrm{cpm}$.

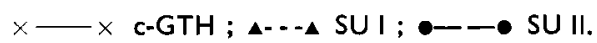

$$
\frac{\mathrm{cpm} \text { lié }}{\mathrm{cpm} \text { total }} \times 100 \pm \text { limites pour } \mathrm{p}=0,05 \text {. }
$$

2. Système IS-SU I ${ }^{125}$ I-SU I (fig. 4). Le pourcentage de liaison en l'absence de la sous-unité (SU I) non marquée est de 49,3 (44,5-54), il est de 43,0 $(39,6-46,4)$ pour $0,01 \mathrm{ng}$ de la SU I. La c-GTH déplace aussi cette liaison, mais la transformation en Logit-Log (dose) montre que les droites ne sont pas parallèles $\left(F_{\text {cov. }}=17,15\right.$ (dl 1-14) $\mathrm{P}<0,001)$. SU II même à des doses élevées (100 ng) n'est pas capable de la déplacer. 


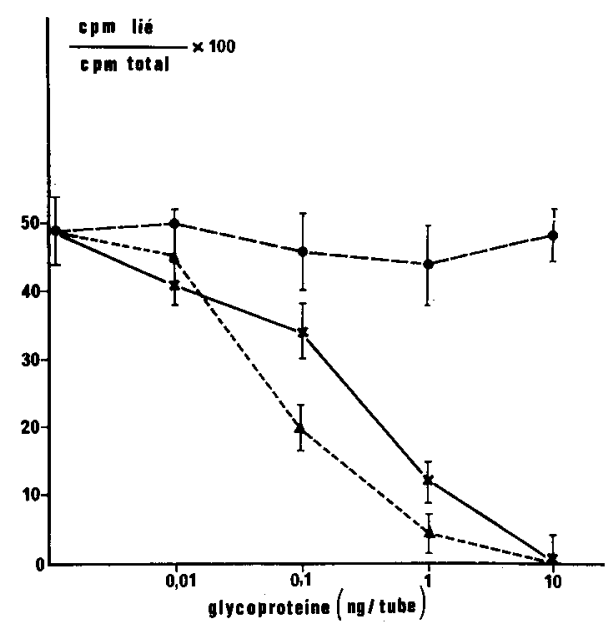

FIG. 4. - Déplacement de la liaison de $125 /$-SU I à IVIS-SU I par c-GTH, SU I ef SUII.

Chaque essai est réalisé en triplica. IS-SU I dilution finale 1/400 000. 125|-SU $1: 13700 \mathrm{cpm}$. $\times — \times$ c-GTH ; $\triangle-. .-$ SUI; •-— SU II.

$$
\text { I } \frac{c p m \text { lié }}{c p m \text { total }} \times 100 \pm \text { limites pour } p=0.05 \text {. }
$$

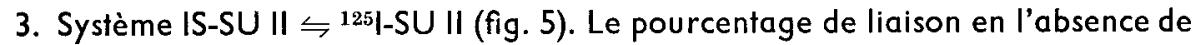
la sous-unité (SU II) non marquée est de 28,6 (24,9-32,3), il est de 18,3 (16,1-20,5) pour $0,01 \mathrm{ng}$ de la SU II. La sensibilité est donc bien inférieure à $10 \mathrm{pg}$. Dans ce système, la

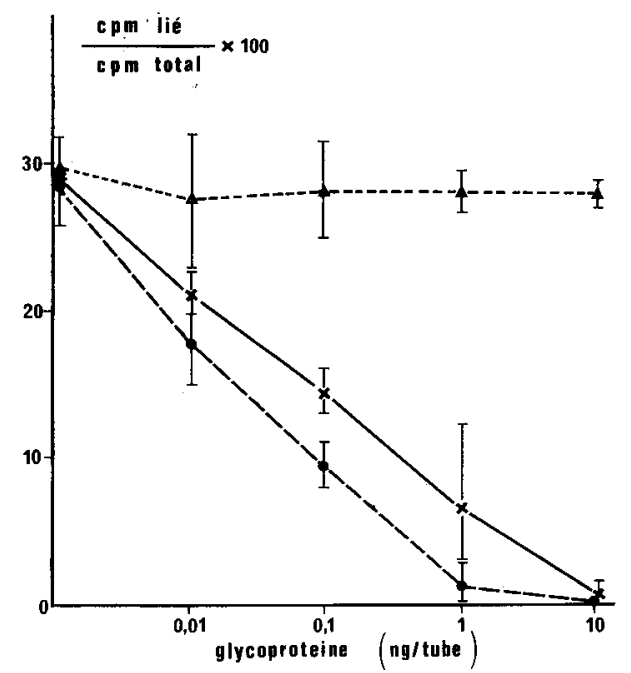

FIG. 5. - Déplacement de la liaison de ${ }^{125} /$-SU II à l'IS-SU II par c-GTH, SU I et SU II. Chaque essai est réalisé en triplica. IS-SU II dilution finale 1/400 000. ${ }^{125}$-SU II : $12250 \mathrm{cpm}$. $\times-\times c-G T H$; - SU II ; a- --a SUI. $\prod \frac{c p m \text { lié }}{c p m \text { total }} \times 100 \pm$ limites pour $p=0,05$. 
c-GTH est également capable de déplacer la liaison. La transformation en Logit-Log (dose) montre que dans ce cas les droites obtenues avec la SU II et la c-GTH marquées sont parallèles $\left(F_{\text {cov }}=3,08(\mathrm{dl} \mathrm{1-14)} p>0,05)\right.$. La réaction croisée est voisine de 20 p. $100 \mathrm{SU}$ I ne la déplace sensiblement pas, même d̀ des doses élevées (100 ng).

Etudiées dans chacun de ces trois systèmes, r-LH, r-FSH, r-TSH et r-PRL ne manifestent aucune réaction croisée, même à des doses élevées (100 ng).

\section{Discussion.}

La c-GTH, SU I et SU II se révèlent être très antigéniques chez le lapin comme le soulignent les titres élevés des immunsérums anti c-GTH, anti SU I et anti SU II chez cette espèce. Un résultat analogue a été observé pour la c-GTH par Breton et al. (1971) chez le cobaye. La sensibilité des trois systèmes radioimmunologiques qui ont été développés ici est élevée.

La technique de marquage à la chloramine $T$ ne modifie apparemment pas les caractéristiques biochimiques (coefficient d'exclusion sur Sephadex G 100, comportement électrophorétique) des sous-unités. Par contre une importante dissociation de la c-GTH en ses sous-unités est observée sous l'influence de l'iodation et les solutions de ${ }^{125} \mid-c-G T H$ sont enrichies en sous-unités marquées. En conséquence, l'affinité de la c-GTH iodée pour l'IS-SU I ef plus particulièrement pour I'IS-SU II peut être surestimée. II en est de même pour la détermination des réactions croisées de SU I ef SU II dans le système IS-c-GTH ${ }^{125 \mid} \mathrm{c}-\mathrm{GTH}$.

Même si elle est surestimée, la réaction croisée entre c-GTH ef SU II est bien démontrée par nos résultats. Dans ces conditions et dans la mesure où la SU II libre serait présente dans le plasma ou l'hypophyse, la détermination radioimmunologique du taux d'hormone proprement dite dans ces matériels, par l'emploi de l'IS-cGTH peut conduire à un résultat inexact. Chez les mammifères et dans certaines conditions physiologiques, il a été montré que les taux plasmatiques en sous-unités libres pouvaient être élevés (Vaiłukaitis, 1977).

En ce qui concerne les poissons téléostéens, la recherche de la présence des sousunités libres dans le plasma n'a pas encore été abordée. D'un point de vue biochimique, nous avons observé que certaines caractéristiques de l'équilibre entre la c-GTH et ses sous-unifés sont différentes de ce qui est observé dans le cas des hormones glycoprotéiques hypophysaires mammaliennes ; la dissociation et l'association sont en particulier beaucoup plus rapides et ceci pourrait avoir une signification physiologique (Fontaine et Burzawa-Gérard, 1978). L'application des résultats présentés dans ce travail ouvre l'accès à ces recherches. Les immunsérums obtenus à partir des sousunités se révèlent être bien plus spécifiques que celui obtenu à partir de la c-GTH. En particulier il n'y a pas de réaction croisée entre les deux sous-unités de la c-GTH. Ce résultat permet donc le dosage sélectif de l'une ou l'autre sous-unité dans la mesure où la c-GTH elle-même est écartée.

Symposium sur la Reproduction des Poissons Paimpont, France, 19-21 septembre 1977.

Remerciements. - Nous exprimons ici nos remerciements à Monsieur Jutisz et à Mme Ribot qui nous ont donné les hormones gonadotropes de rat. Nous remercions également le NIAMD (National Institute of Arthristis and Metabolic Diseases, Rat 
pituitary hormone Program, Bethesda, USA) qui a fourni l'hormone thyréotrope et la prolactine de rat.

\section{Références}

BRETON B., BILLARD R., JALABERT B., 1973. Spécificité d'action et relations immunologiques des hormones gonadotropes de quelques téléostéens. Ann. Biol. anim. Bioch. Biophys., 13, 347-362.

BRETON B., BILLARD R., JALABERT B., KANN G., 1972. Dosage radio-immunologique des gonadotropines plasmatiques chez Carassius auratus, au cours du nycthémère et pendant l'ovulation. Gen. comp. Endocrinol., 18, 463-468.

BRETON B., KANN G., BURZAWA-GERARD E., BILLARD R., 1971. Dosage radioimmunologique d'une hormone gonadotrope de carpe (Cyprinus carpio). C. R. Acad. Sci. Paris, Série D, 276, 715-718.

BURZAWA-GÉRARD E., 1971. Purification d'une hormone gonadotrope hypophysaire de poisson téléostéen, la carpe (Cyprinus carpio L.). Biochimie, 53, 545-552.

BURZAWA-GÉRARD E., 1974. Séparation et réassociation des sous-unités de l'hormone gonadotrope d'un poisson téléostéen, la carpe (Cyprinus carpio), C. R. Acad. Sci. Paris, Série D, 279, $1681-1684$.

BURZAWA-GÉRARD E., KERDELHUÉ B., FONTAINE Y.-A., 1977. Propriétés radioimmunologiques des immun-sérums de l'hormone gonadotrope (c-GTH) de la Carpe (Cyprinus carpio L.) et de ses sous-unités. 9th Conf. Europ. comp. Endocrinol., Giessen, July 31-August 61977.

CRIM L.W., MEYER R. K., DONALDSON E. M., 1973. Radioimmunoassay estimates of plasma gonadotropin levels in the spawning pink salmon. Gen. comp. Endocrinol., 21, 69-76.

FONTAINE Y. A., BURZAWA-GÉRARD E., 1978. Biochemical and biological properties of fish gonadotropins and their subunits. Comparison with mammalian hormones. In MacKERNS K., Structure and function of gonadotropins (sous presse).

GREENWOOD F. C., HUNTER W. M., GLOVER J. S., 1963 . The preparation of 131 -labelled human growth hormone of high specific radio-activity. Biochem. J., 89, 114-123.

KERDELHUÉ B., PITOULIS S., JUTISZ M., 1971. Ełude par radioimmunologie de la spécificité des immunsérums de l'hormone lutéinisante (LH) ovine et de ses sous-unités. C. R. Acad. Sci. Paris, série $D, 273,511-514$.

VAITUKAITIS J. L., 1977. Subunit secretion. in JAMES V. H. T., Endocrinology. Proc. 5th inter. Congr. Endocrinol., 1976, 2, 104-107, Excerpta Med. Publ. 